\title{
Concomitant Prostatic Schistosomiasis and Adenocarcinoma: Case Report and Review
}

Carlos Alberto Basílio-de-Oliveira, Armando Aquino, Eliane Francine Simon and Walter A. Eyer-Silva
University Hospital Gaffrée e Guinle; IASERJ Hospital; Pinheiro Machado Health House, Rio de Janeiro, RJ, Brazil

The term schistosomiasis encompasses a group of infectious disorders caused by five species of the genus Schistosoma, a blood trematode of outstanding importance in tropical areas. Some of these disorders have long been associated with malignant neoplasia, the most striking association being between disease caused by Schistosoma haematobium, the predominant etiological agent of urinary schistosomiasis, and squamous cell carcinoma of the bladder, a relatively uncommon vesical cancer in non-endemic areas. Four cases of simultaneous adenocarcinoma and schistosomiasis of the prostate have been previously reported (S. haematobium in three and $S$. mansoni in one). We report a fifth case of concomitant adenocarcinoma and schistosomiasis of the prostate in a 68-year-old Brazilian patient infected with $S$. mansoni. We also review the medical literature on the association between schistosomiasis and cancer.

Key Words: Adenocarcinoma, prostate, schistosomiasis.

Schistosomiasis is one of the most important tropical infectious diseases in the world. It is estimated that around 200 to 300 million people are infected and an additional 600 million to 1 billion are at risk for acquiring the infection [1]. The term schistosomiasis encompasses a group of acute and chronic clinical and pathological disorders caused by infection with one or more of five species of digenetic worms of the genus Schistosoma. The species of major global importance are Schistosoma mansoni, S. haematobium and $S$. japonicum. In some areas more than one species coexist. The adult worms of these blood flukes inhabit the venous system draining the urinary bladder and the bowel and, in contrast to other human trematodes, have different sexes [2].

Received on 25 May 2001; revised 15 February 2002.

Address for correspondence: Dr. Carlos Alberto Basílio-deOliveira. Serviço de Anatomia Patológica. Hospital Universitário Gaffrée e Guinle. Rua Mariz e Barros, 775, Zip Code: 20270-004 Rio de Janeiro/RJ, Brazil.

The Brazilian Journal of Infectious Diseases 2002;6(1):45-49 (C) 2002 by The Brazilian Journal of Infectious Diseases and Contexto Publishing. All rights reserved. $1413-8670$
Schistosomiasis has long been associated with malignant neoplasia [3-5]. A striking geographical coincidence has been found, for example, between squamous cell carcinoma of the bladder, a relatively uncommon type of vesical cancer, and areas where disease caused by $S$. haematobium is highly prevalent [6,7]. In China, an association between colorectal cancer and longstanding, diffuse, colitis due to $S$. japonicum has been suggested [8-10]. A total of 4 previous cases of prostatic adenocarcinoma (PAC) and concomitant gland schistosomiasis (S. haematobium in 3 and $S$. mansoni in 1) have been described [1113]. We report a fifth case of simultaneous prostatic adenocarcinoma and schistosomiasis in a Brazilian patient infected with S. mansoni.

\section{Case Report}

A 68-year-old white, asymptomatic, patient born in Rio de Janeiro came for routine digital rectal examination. He gave a history of heavy tobacco use for as long as three decades and described himself as a social drinker. He used to work at administrative 
activities in indoor environments. His medical history and general physical examination were unremarkable. Digital rectal examination, however, revealed a welldelimited prostate with a small nodule on the superior part of the left lobe. Laboratory evaluation showed a prostatic specific antigen (PSA) of $9.2 \mathrm{mg} / \mathrm{dl}$ and was otherwise normal. Transrectal biopsy disclosed a PAC and simultaneous infection with $S$. mansoni eggs (Figure 1). The patient informed that he never left the State of Rio de Janeiro and the exposure to cercariae could only be linked to river baths in Nova Iguaçu county.

A radical prostatectomy was then performed. On histopathology the gland was found to be diffusely infiltrated with PAC and scattered schistosomal eggs could be seen. Screening for distant metastatic spread (including a bone cintigraphy) was negative but a right renal mass (subsequently identified as a concomitant renal cell carcinoma) was found. Schistosomiasis was treated with a standard single dose of oxamniquine.

\section{Discussion}

Some infectious diseases are an important preventable cause of human cancer [5]. Among them, schistosomiasis has long been associated with certain forms of malignant disease. The most striking association comes from areas where urinary schistosomiasis is highly prevalent. Urinary schistosomiasis is generally caused by $S$. haematobium. It is the world's leading cause of hematuria and is endemic in many parts of Africa, especially along the Nile Valley, and in the Middle East [2,14]. Male and female adult worms of $S$. haematobium preferentially inhabit the perivesical venous plexus and deposit eggs in the wall of the bladder, or to a lesser extent in the uterus, vaginal wall, and prostate, among other organs. Eggs deposited in the wall of the bladder may break through the lumen and be recovered in the urine. Among 190 consecutive autopsies of patients who died of varying causes at an university hospital in Cairo, 117 patients were diagnosed with $S$. haematobium schistosomiasis and $40.6 \%$ of these were considered to have severe disease leading to major urinary complications such as hydroureter, hydronephrosis and pyelonephritis [15]. An important finding of this study came from the comparison of tissue egg burden between S. haematobium and S. mansoni infection since 51 patients were found to be coinfected. In all organs studied, S. haematobium egg burdens were at least tenfold higher than those of S. mansoni. Exceedingly high mean egg counts per gram of tissue were found in sites such as the bladder $(49,313)$ and the lower right ureter $(43,240)$ in $S$. haematobium schistosomiasis.

There is a geographical coincidence between bladder cancer and urinary schistosomiasis in some highly endemic areas of Africa and the Middle East. In 1911, Ferguson published a case series of 40 Egyptian patients with simultaneous bladder cancer and $S$. haematobium infection and thought that malignant transformation was triggered by prolonged irritation of the epithelium lining the bladder by the passage and entrapment of ova [3]. El-Bolkainy et al. reported on a series of 1095 bladder cancer cases treated by radical cystectomy from 3 treatment facilities in India between 1976 and 1978 [6]. Most specimens (82.4\%) had demonstrable schistosome eggs. In egg-positive cases the tumors developed at a younger age ( 46.7 years) than in egg-negative ones (53.2 years). Also, among egg-positive cases squamous cell carcinoma, a relatively uncommon tumor in non-endemic areas, predominated instead of transitional cell (urothelial) carcinoma and adenocarcinoma. Malik et al. [7] studied 255 bladder carcinoma cases from Sudan and found $122(47.9 \%)$ cases of transitional cell carcinomas, 101 (39.6\%) of squamous cell carcinomas, $26(10.2 \%)$ of anaplastic carcinomas and $6(2.3 \%)$ of adenocarcinomas. Associated urinary schistosomiasis was found in $43.9 \%$ of squamous carcinomas, $11.8 \%$ of anaplastic carcinomas and only $3.2 \%$ of the transitional cell carcinoma cases. The authors reviewed the available data on the frequency of squamous carcinoma among total bladder cancer cases and found figures of $62.3 \%, 39.7 \%$ and $1.6 \%$ for Egypt, Sudan and England, respectively. Other authors, however, failed to find an association between urinary schistosomiasis and bladder cancer $[15,16]$. A potential methodological weakness from the Cairo university 
Figure 1. Histopathological study of a tissue fragment obtained through a transrectal biopsy reveals a moderately differentiated prostatic adenocarcinoma seen as a malignant proliferation of acinar structures with hyperchromatic areas. An egg of S. mansoni with its lateral spine is also seen (x160; hematoxycilin and eosin)

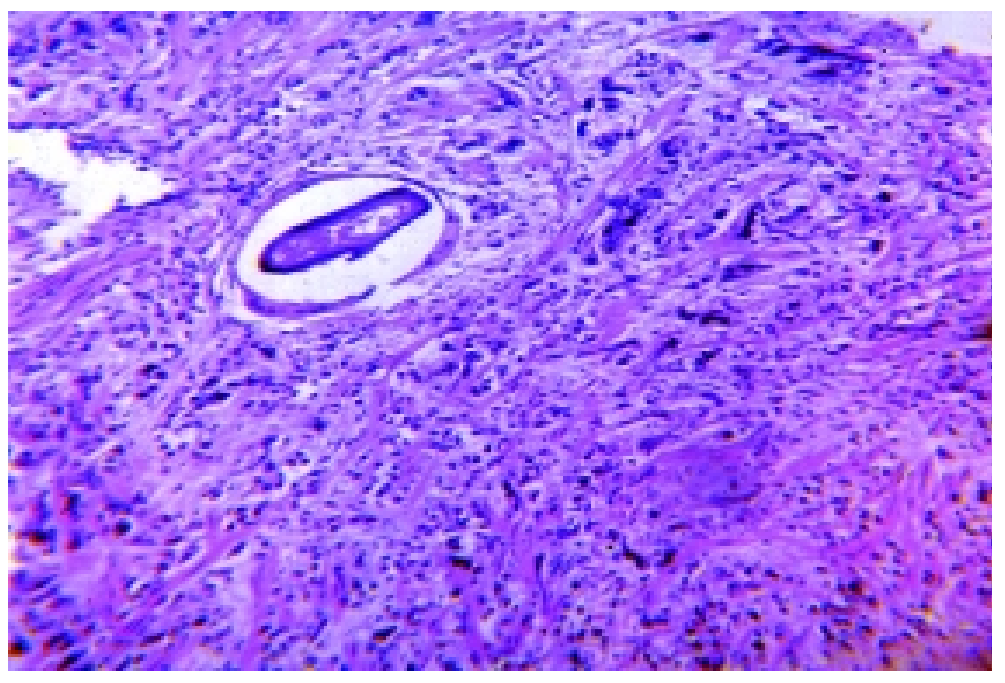

hospital consecutive autopsy study [15] was selection bias due to the existence of a cancer hospital in town. It has been suggested that nitrosamine carcinogens, produced by nitrate-reducing bacteria found in high concentration and at a greater frequency in the urine of patients with schistosomiasis, preferentially affects portions of the urinary tract into which calcified eggs or other foreign particulate matter have been injected [14].

An association between schistosomiasis due to $S$. japonicum and carcinoma of the colon and rectum has been suggested. Male and female adult worms of $S$. japonicum have a predilection for the branches of the inferior mesenteric and superior haemorrhoidal veins, but the superior mesenteries and the caval system may also be invaded $[2,17]$. In China, colorectal cancer achieves relatively high incidence rates in areas endemic for schistosomiasis. On the basis of their observations of 90 cases of simultaneous colorectal carcinoma and schistosomiasis diagnosed between 1951 and 1964, Ming-Chai et al. proposed that, in its late phases, chronic colitis due to $S$. japonicum is a premalignant condition not infrequently leading to cancer [8]. Their patients had a mean age of only 36.9 years (range, 2063), most (79) were rice-growing farmers and $10 \mathrm{had}$ multicentric carcinoma. The same group later reported on a pathological study of colorectal carcinoma colectomy specimens [9]. Among 454 specimens, 289 $(63.6 \%)$ were associated with schistosomiasis and 165 were not. They found that specimens from patients with schistosomiasis had granulomatous-associated changes of polyp formation, ectopically proliferating glands and ulceration that could well be predisposing abnormalities leading to the development of cancer in a manner analogous to that in ulcerative colitis. Patients with schistosoma-associated neoplasia were a mean of 6.5 years younger and were more likely to have welldifferentiated tumors than patients without schistosomiasis. A common feature in schistosomaassociated cases was the widespread colonic infection and a long history of colitic symptoms [9].

Schistosomiasis of the prostate is poorly documented in the medical literature. In the Cairo university hospital consecutive autopsy study the investigators found in prostates from patients with 
haematobium and mansoni schistosomiasis a mean of 8,058 and 11 eggs per gram of prostatic tissue, respectively [15]. They found 3 cases of PAC among the 190 studied patients: 2 in the schistosomiasis and 1 in the control group. They failed, however, to specify whether the prostates had concomitant schistosomal infection. In Zambia, a consecutive autopsy study of 50 patients who died of varying causes found that $62 \%$ of the bladders, $58 \%$ of the seminal vesicles and $50 \%$ of the prostates were infected with $S$. haematobium eggs [18]. No cases of mansoni schistosomiasis were found. No major structural prostatic derangements were described. A Spanish study of transrectal ultrasound abnormalities among 9 young returning travelers who complained of hemospermia and perineal discomfort and who were diagnosed with schistosomiasis (5 with $S$. haematobium, 2 with $S$. intercalatum, 2 with $S$. mansoni and 1 with a mixed S. mansoni-S. haematobium infection) found that 7 patients had prostatic calcifications and 2 had an enlarged prostate. The investigators found that urine and semen were useful specimens for a parasitological diagnosis of male genital schistosomiasis [19].

Four previously reported cases of concomitant PAC and schistosomiasis were recovered through MEDLINE search [11-13]. One case of simultaneous PAC and S. mansoni gland infection was diagnosed in a 49-year-old Puerto Rican-born patient living in the United States for 25 years (this case has been published twice; $[11,12])$. The patient had a diffusely indurated prostate with evidence of widespread bone metastasis. A biopsy specimen showed a diffusely infiltrating adenocarcinoma containing $S$. mansoni eggs surrounded by acute and chronic inflamatory cells with epithelioid granuloma. Outstanding features of the case were the relatively young age at the time of diagnosis (49 years) and the heavy gland infection by $S$. mansoni eggs. An intriguing South African case series was reported by Cohen et al. [13]. They described 3 patients seen within a 7-month period in a rural endemic area who developed cancer of the prostate at an extremely young age (1 patient was 27 and the other 2 were 29-years-old). All had elevated PSA levels with advanced malignant disease and simultaneous florid gland infection. An adult worm could also be seen in a tissue section from one of the patients. Canadian pathologists reported still another patient with simultaneous PAC and genital schistosomiasis: a 55year-old Ghanian residing in Canada for 19 years who had been treated for PAC 2 years before. When submitted to a new series of directed biopsies of the prostate and seminal vesicles due to increasing PSA levels, an extensive malignant infiltration of the prostate, peri-prostatic fibroadipose tissue and seminal vesicles was demonstrated. In addition, abundant $S$. haematobium eggs were seen in the seminal vesicles, but not in the prostate, biopsies [20].

In summary, we report a fifth case of simultaneous PAC and schistosomiasis (the second case associated with $S$. mansoni). In contrast to the previous reports, our patient's malignant disease was diagnosed at an older age. Whether the gland infection contributed to the development of the malignant prostatic neoplasia is not known. However, an epidemiological link between these 2 disorders remains limited to a few case reports of simultaneous disease. Although the South African series [10] reported malignant disease at an unusually young age in conjunction with extremely heavy gland infection, a true cause and effect relationship should lead to stronger epidemiological data. This would be expected especially from areas where $S$. haematobium is highly endemic, since it is associated with a substantially high prostate egg burden [15]. We believe that the current data only allow one to consider these isolated cases as incident simultaneous findings of two common, unrelated, disorders.

\section{Acknowledgement}

We are indebted to Prof. Maria José Conceição, from the Laboratório de Controle da Esquistossomose at Fundação Oswaldo Cruz, Rio de Janeiro, for critically reviewing the manuscript. 


\section{References}

1. King C.H., Mahmoud A.A.F. Schistosoma and other trematodes. In: Gorbarch S.L., Bartlett J.G., Blacklow N.R., eds. Infectious Diseases. Philadelphia: W.B. Saunders Company, 1998.

2. Markell E.K., John D.T., Krotoski W.A. Markell and Voge's Medical Parasitology. Philadelphia, USA. W.B. Saunders Company, 1999.

3. Ferguson A.R. Associated bilharziosis and primary malignant disease of the urinary bladder, with observations on a series of forty cases. Journal of Pathology and Bacteriology 1911;16:76-94.

4. Cheever A.W. Schistosomiasis and neoplasia. J Natl Cancer Inst 1978;61:13-8.

5. Kuper H., Adami H-O., Trichopoulos D. Infections as a major cause of human cancer. J Intern Med 2000;248:171-83.

6. El-Bolkainy M.N., Mokhtar N.M., Ghoneim M.A., Hussein M.H. The impact of schistosomiasis on the pathobiology of bladder carcinoma. Cancer 1981;48:2643-8.

7. Malik M.O.A., Veress B., Daoud E.H., el Hassan A.M. Pattern of bladder cancer in the Sudan and its relation to schistosomiasis: a study of 255 vesical carcinomas. J Trop Med Hyg 1975;78:219-26.

8. Ming-Chai C., Jen-Chun H., Pei-Yu C., et al. Pathogenesis of carcinoma of the colon and rectum in schistosomiasis japonica. Chinese Med J 1965;84: 513-25.

9. Ming-Chai C., Chi-Yuan C., Pei-Yu C., Jen-Chun H. Evolution of colorectal cancer in schistosomiasis. Cancer 1980;46:1661-75.

10. Ming-Chai C., Pei-Yu C., Chi-Yuan C., et al. Colorectal cancer and schistosomiasis. Lancet 1981;1:971-3.

11. Alexis R., Domingo J. Schistosomiasis and adenocarcinoma of prostate: a morphologic study. Hum Pathol 1986; $17: 757-60$.

12. Godec C.J., Grunberger I., Carr G.A. Simultaneous presence of schistosomiasis and advanced cancer in prostate. Urology 1992;39:547-9.

13. Cohen R.J., Edgar S.G., Cooper K. Schistosomiasis and prostate cancer. Pathology 1995;27:115-6.

14. Smith J.H., Christie J.D. The pathobiology os Schistosoma haematobium infection in humans. Hum Pathol 1986; $17: 333-45$.

15. Smith J.H., Kamel I.A., Elwi A., von Lichtenberg F. A quantitative post mortem analysis of urinary schistosomiasis in Egypt. Am J Trop Med Hyg 1974;23:1054-71.

16. Attah E.B., Nkposong E.O. Schistosomiasis and carcinoma of the bladder: a critical appraisal of causal relationship. Trop Geogr Med 1976;28:268-72.
17. Chen M.G. Relative distribution of Schistosoma japonicum eggs in the intestine of man: a subject of inconsistency. Acta Trop 1991;48:163-71.

18. Patil P.S., Elem B. Schistosomiasis of the prostate and the seminal vesicles: observations in Zambia. J Trop Med Hyg 1988;91:245-8.

19. Vilana R., Corachán M., Gascón J., Valls E., Bru C. Schistosomiasis of the male genital tract: transrectal sonographic findings. J Urol 1997; 158:1491-3.

20. Ma T.K.F., Srigley J.R. Adenocarcinoma of prostate and schistosomiasis: a rare association. Histopathology 1995;27:187-9. 